\title{
MODIFICAÇÃO QUÍMICA DO MESOCARPO DO COCO BABAÇU COM O POLÍMERO TETRAMETIL-2,6- BROMOIONENO PARA ADSORÇAO DO PESTICIDA PARATION METÍLICO
}

\author{
M. R. A. SOUSA Jr. ${ }^{1}$, A. T. da SILVA ${ }^{1}$, A. A. SANTANA ${ }^{2}$, W. F. de LA SALLES ${ }^{3}$ e A. P. \\ da COSTA FILHO ${ }^{3}$
}

${ }^{1}$ Bolsista de Iniciação Científica da UFMA, discente do curso de Engenharia Química

${ }^{2}$ Professor da Coordenação de Engenharia Química da UFMA

${ }^{3}$ Professor do Departamento de Tecnologia Química da UFMA

E-mail para contato: mauroromeroasj@gmail.com

\begin{abstract}
RESUMO - A preocupação com a contaminação de sistemas aquáticos superficiais e subterrâneos por pesticidas tem crescido no meio científico. Dentre os pesticidas mais utilizados para tal finalidade, encontra-se o paration metílico (inseticida organofosforado) que é amplamente utilizado para controle de parasitas e larvas nas lavouras. O objetivo geral dessa pesquisa foi utilizar o mesocarpo de babaçu como matriz para ancoragem do polímero catiônico tetrametil-2,6-bromoioneno nas proporções de 2, 4, 6 e $8 \mathrm{~g}$ de polímero para $20 \mathrm{~g}$ deste material com a finalidade de modificar a polaridade do mesocarpo, com vistas na sua aplicação como adsorvente para pesticidas em ambientes aquáticos, em especial, o paration metílico. Para a caracterização e sintetização do mesocarpo de babaçu modificado com o polímero quaternário foram feitas a capacidade adsortiva deste novo adsorvente utilizando a Cromatografia a Líquido de Alto Desempenho (HPLC), Espectroscopia de Absorção na Região do Infravermelho com Transformada de Fourrier (FTIR), Ressonância Magnética Nuclear de Hidrogênio (RMN-H) e Microscopia Eletrônica de Varredura (MEV). Os resultados para as análises das amostras de mesocarpo de babaçu modificadas mostraram que o polímero interagiu com o mesocarpo provocando alterações microestruturais na matriz (mesocarpo de babaçu) bem como houve adsorção do pesticida na matriz de mesocarpo modificada com percentuais diferentes de polímero catiônico.
\end{abstract}

\section{INTRODUÇÃO}

A preocupação com a contaminação de sistemas aquáticos superficiais e subterrâneos por pesticidas tem crescido no meio científico. Estudos desenvolvidos em várias regiões do mundo têm mostrado que a porcentagem dos produtos utilizados na agricultura que atingem os ambientes aquáticos é geralmente baixa. Entretanto, pesticidas persistentes e com grande mobilidade no ambiente têm sido detectados em águas superficiais e subterrâneas. Dentre os pesticidas mais estudados, atualmente, destaca-se o paration metílico, um inseticida 
organofosforado muito utilizado na agricultura e também na aquicultura para o controle de pragas (Rodrigues et al., 1997).

Dentre os materiais mais abundantes no Maranhão, destaca-se o babaçu que vem sendo amplamente comercializado no país (cerca de $70 \%$ da produção nacional) (Cruz e Coelho, 2006). Em relação ao babaçu, o seu mesocarpo, rico em macromoléculas que possuem sítios ativos para interação química, dentre eles os grupos hidroxilas das unidades de glicose, têm despontado como uma fonte rica em polímeros naturais que podem ser modificados quimicamente para aplicações em diversas áreas, em especial como adsorvente de pesticida.

Neste contexto, o objetivo principal deste trabalho foi utilizar o mesocarpo de babaçu como matriz para ancoragem do polímero catiônico tetrametil-2,6-bromoioneno com a finalidade de interagir as cargas positivas inerentes ao policátion com os grupos hidroxilas gerando um material reticulado. Além disso, a utilização de polímero catiônico contendo 6 grupos metilênicos como espaçador da cadeia polimérica teve como objetivo modificar a polaridade do mesocarpo, tornando-o moderadamente organofílico, com vistas na sua aplicação como adsorvente para pesticidas em ambientes aquáticos, em especial, o paration metílico.

\section{EXPERIMENTAL}

\subsection{Material}

A farinha do mesocarpo de babaçu foi fornecida pela fábrica Florestas Brasileiras S.A., localizada no município de Itapecuru-Mirim, no estado do Maranhão, serviu como matériaprima neste estudo, a qual foi modificada quimicamente para ter aplicação como agente adsorvente. Esta farinha foi obtida por meio de processo de classificação e moagem do mesocarpo como parte do aproveitamento integral do coco (in natura), apresentando uma granulometria de 150 a $180 \mu \mathrm{m}$.

Síntese do polímero: O polímero tetrametil-2,6-bromoioneno foi sintetizado de acordo a reação de Menshutkin, conforme apresentou (Costa Filho, 2005). A reação ocorre a partir dos monômeros N,N,N',N'Tetrametiletilenodiamina (TMEDA) e 1,6-dibromohexano em quantidades equimolares sob agitação à temperatura ambiente, durante cerca de 168 horas, utilizando-se como solvente reacional a dimetilformamida (DMF). Ao término da reação, adicionou-se um excesso de acetona e em seguida fez-se a filtração a vácuo utilizando-se funil sinterizado. O polímero obtido foi seco em estufa à vácuo por 48 horas.

\subsection{Métodos}

Modificacão Do Mesocarpo De Babacu Com o Polímero 2,6-Bromoioneno: O mesocarpo do babaçu foi modificado com o polímero catiônico nas proporções de $2,4,6$ e $8 \mathrm{~g}$ de polímero/ $20 \mathrm{~g}$ de mesocarpo de babaçu. As amostras foram preparadas em erlenmeyers 
contendo as quantidades de polímero previamente pesadas e dissolvidas em $100 \mathrm{ml}$ de água. Em cada uma destas soluções foi adicionada $20 \mathrm{~g}$ de mesocarpo de babaçu mantido à temperatura ambiente sob agitação por 24 horas. Em seguida, as amostras foram filtradas sob vácuo em funil de Buchner, lavadas com água destilada durante a filtração e posteriormente transferidas para placas de Petri e mantidas em estufa a $70{ }^{\circ} \mathrm{C}$ por 24 horas para secagem. Cada amostra seca foi macerada usando-se gral e pistilo.

Caracterizacão Do Mesocarpo De Babaçu Modificado Com o Polímero Tetrametil2,6-Bromoioneno: As amostras de mesocarpo de babaçu modificadas com o polímero tetrametil-2,6-bromoioneno foram caracterizadas por FTIR, a fim de verificar as bandas de absorção características do polímero e confirmar a sua presença no compósito com o mesocarpo de babaçu. Além disso, as amostras de mesocarpo de babaçu modificadas com o polímero tetrametil 2,6-bromoioneno foram analisadas por MEV a fim de se observar mudanças morfológicas.

Avaliação Da Capacidade Absortiva Do Mesocarpo De Babacu Modificado Com o Polímero Tetrametil-2,6-Bromoioneno Frente Ao Pesticida Paration Metílico: Para realização experimental da avaliação da capacidade adsortiva do mesocarpo modificado com o polímero frente ao pesticida paration metílico utilizou-se a HPLC.

\section{RESULTADOS E DISCUSSÃO}

\subsection{Síntese e caracterização do polímero tetrametil-2,6-bromoioneno}

A síntese do polímero tetrametil-2,6-bromoioneno obteve um rendimento acima de 95\%. Os resultados obtidos através de FTIR demonstraram o mesmo comportamento relatado por (Costa Filho, 2005), mostrando a existência de bandas de vibração relacionadas à absorção de água por parte do polímero bem como deformações axiais e angulares nos grupos $\mathrm{CH}_{2}$ e $\mathrm{CH}_{3}$ e estiramentos nas ligações $\mathrm{C}-\mathrm{C}$ e N ${ }^{+}-\mathrm{CH}_{2}$. Na análise de $\mathrm{RMN}-\mathrm{H}$, os resultados obtidos são compatíveis com aqueles apresentados por (Silverstein et al., 2010), onde é possível observar o deslocamento químico referente ao grupo quaternário de amônio assim como modificações ocasionadas pelos hidrogênios dos grupos metilas ligados aos nitrogênios quaternários.

\subsection{Caracterização das amostras de mesocarpo modificado através de espectroscopia de absorção na região do infravermelho com transformada de fourrier-FTIR}

A Figura 1 apresenta os espectros do mesocarpo de babaçu puro e aqueles modificados com polímero 2,6-bromoioneno nas proporções de 2, 4, 6 e 8 gramas de polímero, respectivamente. 
Figura 1 - Espectro de absorção na região do Infravermelho do mesocarpo do babaçu puro e modificado.

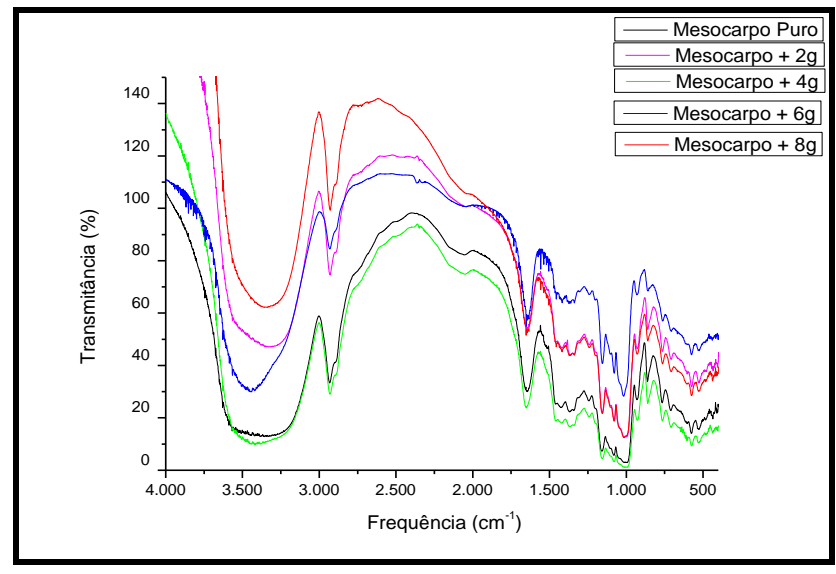

Observando-se os espectros do mesocarpo puro com os modificados, pode-se observar o aumento da intensidade das bandas características relacionadas à $\mathrm{CH}_{2}$ e $\mathrm{CH}_{3}$ em aproximadamente $2930 \mathrm{~cm}^{-1}$ e $1365 \mathrm{~cm}^{-1}$, respectivamente, as quais estão associadas a quantidades crescentes de polímero na matriz de mesocarpo.

\subsection{Caracterização do mesocarpo de babaçu modificado e não modificado com o polímero através da MEV}

Os resultados obtidos por MEV para o mesocarpo de babaçu não modificado e modificado com polímero (proporção de $8 \mathrm{~g}$ de polímero para $20 \mathrm{~g}$ de mesocarpo) estão mostrados na Figura 2.

Figura 2 - MEV do mesocarpo do coco babaçu in natura (a) e modificado com o polímero catiônico 2,6-bromoioneno (b) ampliado 1500 vezes (aproximadamente $181 \mu \mathrm{m}$ ).

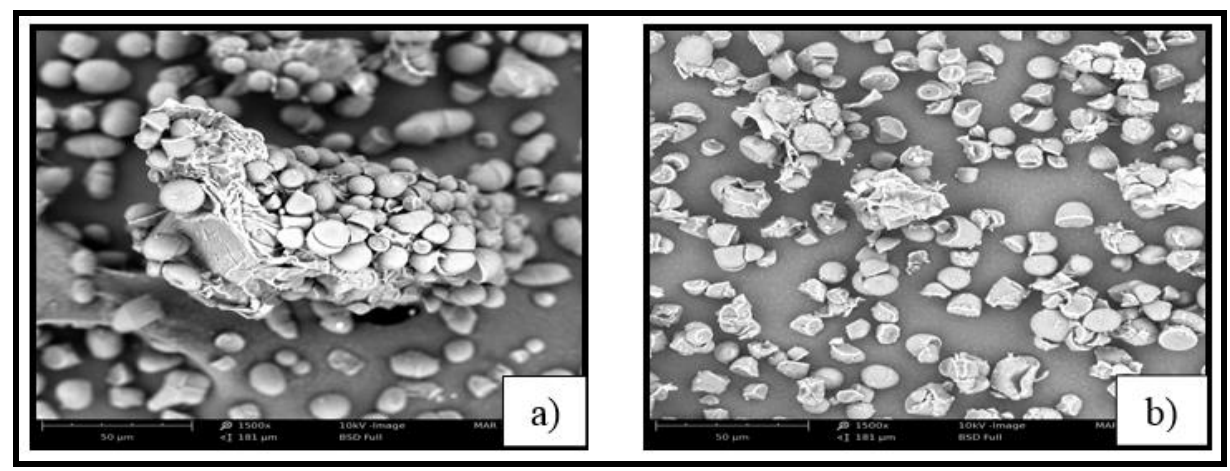

Com base nas observações realizadas na Figura 2, pode-se constatar que houve a quebra da matriz inicial que aglomerava as partículas (provavelmente cadeias de amido), além de uma redução significativa, em aproximadamente metade, do tamanho original da partícula. Uma possível explicação para tal resultado pode estar relacionado com a massa molar do polímero ser significativamente menor que a massa molar do amido presente no mesocarpo, 
pois o amido é constituído por unidades de glicose unidas por ligações $\alpha-1,4$ e apresenta uma estrutura granular semicristalina (grânulos constituídos por macromoléculas de amilose e amilopectina) (Kok, 2004). Assim, sugere-se que o tamanho das cadeias do polímero catiônico não seja suficientemente grande para interagir com muitas cadeias de amido, simultaneamente, resultando em partículas de tamanho menor. Além disso, realizou-se, também, análise por EDS cujos resultados são apresentados na Tabela 1.

Tabela 1 - Porcentagem dos principais elementos presentes no mesocarpo do coco babaçu puro e modificado com polímero através do EDS

\begin{tabular}{|c|c|c|c|c|}
\hline \multirow{2}{*}{ Amostra analisada } & \multicolumn{4}{|c|}{ Elementos detectados (\%) } \\
\cline { 2 - 5 } & $\mathbf{O}$ & $\mathbf{C}$ & $\mathbf{N}$ & $\mathbf{B r}$ \\
\hline Mesocarpo do coco babaçu puro & 70 & 30 & - & - \\
\hline Mesocarpo do coco babaçu modificado com polímero & 66,7 & 29,3 & 3,4 & 0,6 \\
\hline
\end{tabular}

A Tabela 1 mostra que existe uma grande quantidade de carbono (30\%) e oxigênio (70\%) presentes no mesocarpo do babaçu puro, provenientes, principalmente, do amido e da lignina-celulose. Entretanto, ao se adicionar o polímero catiônico ao mesocarpo do coco babaçu, pode-se constatar um pequeno decréscimo dos elementos oxigênio $(66,7 \%)$ e carbono $(29,3 \%)$ e aparecimento dos elementos nitrogênio $(3,4 \%)$ e bromo $(0,6 \%)$, propondo-se que houve a inserção desse polímero na matriz. Vale-se ressaltar que a baixa quantidade presente do elemento bromo, contra-íons, pode ser interpretada pelos sítios quaternários de amônio que não interagiram e, portanto, continuaram ativos.

\subsection{Testes de adsorção}

Para realização dos testes de adsorção, preparou-se, inicialmente, uma solução do branco a partir de 20 microlitros da solução do paration metílico diluídos em $10 \mathrm{ml}$ de água (concentração de $0,002 \mathrm{ppm}$ ) onde o pico relativo ao pesticida foi verificado próximo a 6 minutos e, um segundo pico, em 8 minutos. Assim, para verificar a capacidade adsortiva das amostras de mesocarpo de babaçu puro e modificados, realizou-se o mesmo procedimento feito para o branco. Os resultados são apresentados na Figura 3.

Figura 3 - Cromatograma do branco (pesticida) (a), das amostras de mesocarpo de babaçu puro (b) e modificado com $2 \mathrm{~g}$ (c), $4 \mathrm{~g}$ (d) e $6 \mathrm{~g}$ (e) de polímero catiônico testados como adsorvente do paration metílico.

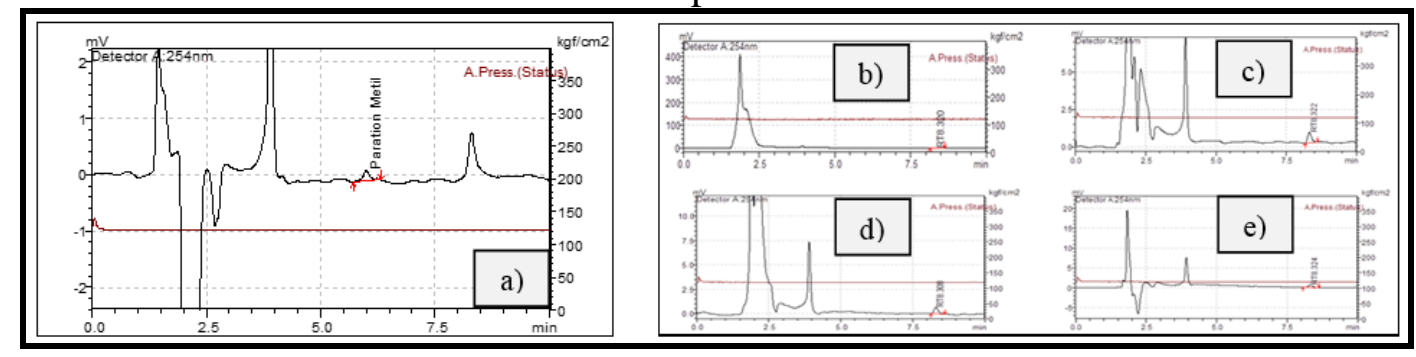

Observa-se que para o mesocarpo puro, o pico referente ao paration metílico desapareceu, sugerindo a adsorção do pesticida por este material. Este mesmo comportamento 
foi observado para os testes realizados com as amostras de mesocarpo de babaçu modificados. Observa-se, também, a diminuição significativa da intensidade do pico em aproximadamente 8 minutos para o mesocarpo puro bem como a diminuição gradativa deste mesmo pico com o aumento da quantidade de polímero nas amostras, indicando que este pico se refere a algum intermediário químico do paration metílico.

\section{CONCLUSÃO}

Os resultados obtidos neste trabalho mostraram que a síntese do polímero tetrametil2,6-bromoioneno teve êxito com rendimento de $95 \%$ e ambas as técnicas, FTIR e RMN-H, confirmaram a sua estrutura. Os ensaios realizados por MEV indicaram a ancoragem do polímero, apresentando uma organização morfológica nas amostras de mesocarpo modificadas com o polímero quando comparadas com a amostra do mesocarpo puro. Apesar dos resultados ainda serem preliminares, pode-se observar a adsorção entre as moléculas do paration metílico e as matrizes de mesocarpo pura e modificadas com o polímero catiônico.

\section{REFERÊNCIAS}

COSTA FILHO, A. P. Síntese e caracterização de nanocompósitos bentonita-ionenos alifáticos com diferentes tamanhos de espaçadores na cadeia polimérica. 2005. $276 \mathrm{f}$. Tese (Doutorado em Ciência e Tecnologia de Polímeros) - Universidade Federal do Rio de Janeiro, Rio de Janeiro, 2005.

CRUZ, G. C. N.; COELHO, M. V. Characterization of the Ca-ATPase activity of Pachymerus nucleorum (Coleoptera: Chysomelidae: Bruchinae) larvae. 2006. Dissertação (Mestrado em Genética e Bioquímica) - Universidade Federal de Urberlândia, Uberlância, 2006. (Submetido para Comparative Biochemistry and Physiology. Part B).

KOK, W. TH. Journal of Cromatrography, 2004.

RODRIGUES, E. L.; PAIVA, M. J. T. R.; PACHECO, F. J. VEIGA, M. L. Efeito agudo do organofosforado Dipterex 500 (Trichlorfon) em baço de curimbatá Prochilodus scrofa (Steindachner, 1881). B. Inst. Pesca, São Paulo, 24 (n. especial), p. 197-203. Disponível em: <http://pesquisa.bvsalud.org/unifesp/resources/prod-117396>. Acesso em: 01 jan. 2017.

SILVERSTEIN, R. M.; WEBSTER, F. X.; KIEMLE, D. J. Identificação Espectrométrica de Compostos Orgânicos. São Paulo: LTC, 2010. 